Bull. Korean Math. Soc. 50 (2013), No. 4, pp. 1357-1365

http://dx.doi.org/10.4134/BKMS.2013.50.4.1357

\title{
CLASS NUMBER DIVISIBILITY OF QUADRATIC FUNCTION FIELDS IN EVEN CHARACTERISTIC
}

\author{
Sunghan Bae And Hwanyup Jung
}

\begin{abstract}
We find a lower bound on the number of real/inert imaginary/ramified imaginary quadratic extensions of the function field $\mathbb{F}_{q}(t)$ whose ideal class groups have an element of a fixed order, where $q$ is a power of 2 .
\end{abstract}

\section{Introduction}

Let $k=\mathbb{F}_{q}(t)$ be the rational function field over the finite field $\mathbb{F}_{q}$ and $\mathbb{A}=\mathbb{F}_{q}[t]$. Let $\infty$ be the infinite place of $k$ associated to $(1 / t)$. Throughout the paper, by a quadratic function field, we always mean a quadratic extension of $k$. A quadratic function field $F$ is said to be real if $\infty$ splits in $F$, and imaginary otherwise. Assume that $q$ is odd. Then any quadratic function field $F$ can be written as $F=k(\sqrt{D})$, where $D$ is a square-free polynomial in $\mathbb{A}$. Let $\mathcal{O}_{F}$ be the integral closure of $\mathbb{A}$ in $F$. In [2], Murty and Cardon proved that there are $\gg q^{\ell\left(\frac{1}{2}+\frac{1}{g}\right)}$ imaginary quadratic function fields $F=k(\sqrt{D})$ such that $\operatorname{deg} D \leq \ell$ and the ideal class group of $\mathcal{O}_{F}$ has an element of order $g$. This result is the function field analogue of the result of Murty for imaginary quadratic fields ([5]). In [4], Friesen proved the existence of infinitely many real quadratic function fields $F$ whose ideal class numbers are divisible by a given positive integer $g$. In [3], using the Friesen's result, Chakraborty and Mukhopadhyay proved that there are $\gg q^{\frac{\ell}{2 g}}$ real quadratic function fields $F=k(\sqrt{D})$ such that $\operatorname{deg} D \leq \ell$ and the ideal class group of $\mathcal{O}_{F}$ has an element of order $g$.

The aim of this paper is to study the same problem in even characteristic case. Assume that $q$ is a power of 2 . Then any quadratic function field $F$ of $k$ can be written as $F=k(y)$, where $y$ is a zero of $\mathbf{x}^{2}+A \mathbf{x}+B=0$ with $A, B \in \mathbb{A}$. Here, we can always assume that $A$ is monic and $(A, B)$ satisfies

Received October 26, 2012.

2010 Mathematics Subject Classification. 11R18, 11R29, 11R58.

Key words and phrases. class number divisibility, quadratic function field, even characteristic.

This work was supported by the National Research Foundation of Korea(NRF) grant funded by the Korea government(MEST)(No. 2012-0000798). 
some property, so that we have $\mathcal{O}_{F}=\mathbb{A}[y]$ and $A$ is uniquely determined since the discriminant of $F$ over $k$ is $A^{2}$ (see $\S 2$, Lemma 2.1). Write $d(F)=\operatorname{deg} A$.

We now state the results of this paper.

Theorem 1.1. Let $q$ be a power of 2 , and let $g$ be a fixed positive integer $\geq 2$. Then there are $\gg q^{\nu(g, \ell)}$ real quadratic function fields $F$ of $k=\mathbb{F}_{q}(t)$ such that $d(F) \leq \ell$ and the ideal class group of $\mathcal{O}_{F}$ contains an element of order $g$, where $\nu(g, \ell)$ is $\frac{\ell}{2 g}$ or $\frac{\ell}{g+1}$ according as $g$ is odd or even.

An imaginary quadratic function field $F$ of $k$ is said to be inert or ramified according as $\infty$ inerts or ramifies in $F$.

Theorem 1.2. Let $q$ be a power of 2 , and let $g$ be a fixed positive integer $\geq 2$. Then there are $\gg q^{\frac{\ell}{g}}$ inert imaginary quadratic function fields $F$ of $k=\mathbb{F}_{q}(t)$ such that $d(F) \leq \ell$ and the ideal class group of $\mathcal{O}_{F}$ contains an element of order $g$.

Theorem 1.3. Let $q$ be a power of 2 , and let $g$ be a fixed positive integer $\geq 2$. Then there are $\gg q^{\frac{\ell}{g-1}}$ ramified imaginary quadratic function fields $F$ of $k=\mathbb{F}_{q}(t)$ such that $d(F) \leq \ell$ and the ideal class group of $\mathcal{O}_{F}$ contains an element of order $g$.

\section{Preliminaries}

Let $q$ be a power of 2 , and $\mathbb{F}_{q}$ be the finite field of $q$ elements. Let $k=$ $\mathbb{F}_{q}(t), \mathbb{A}=\mathbb{F}_{q}[t], \infty$ be the infinite place of $k$ associated to $(1 / t)$ and $k_{\infty}=$ $\mathbb{F}_{q}((1 / t))$ be the completion of $k$ at $\infty$. For $0 \neq A \in \mathbb{A}$, let $\operatorname{sgn}(A)$ be the leading coefficient of $A$.

Let $\Omega$ be the set of pairs $(A, B) \in \mathbb{A} \times \mathbb{A}$ such that $A$ is monic and $(A, B)$ satisfies the property that for any irreducible polynomial $P \operatorname{dividing} A$, the congruence

$$
\mathbf{x}^{2}+A \mathbf{x}+B \equiv 0 \bmod P^{2}
$$

is not solvable in $\mathbb{A}$. Then any quadratic function field $F$ of $k$ can be written as $F=k(y)$, where $y$ is a zero of $\mathbf{x}^{2}+A \mathbf{x}+B=0$ with $(A, B) \in \Omega([6, \S 1])$.

The following lemma is due to Bae (the proof of Lemma 5.1 in [1] given there for real quadratic extension of $k$ is easily seen to be valid for arbitrary quadratic extension of $k$ ).

Lemma 2.1. Let $F=k(y)$ be a quadratic extension of $k$, where $y$ is a zero of $\mathbf{x}^{2}+A \mathbf{x}+B=0$ with $(A, B) \in \Omega$. Let $\mathcal{O}_{F}$ be the integral closure of $\mathbb{A}$ in $F$. Then we have

(i) $\mathcal{O}_{F}=\mathbb{A}[y]$.

(ii) A prime $P$ of $\mathbb{A}$ is ramified in $F$ if and only if $P$ divides $A$. In fact, the discriminant of $F$ over $k$ is $A^{2}$. 
It is easy to see that if $(A, B) \in \Omega$, then $\left(A, C^{2}+A C+B\right) \in \Omega$ for any $C \in \mathbb{A}$. If $F=k(y)=k\left(y^{\prime}\right)$, where $y^{\prime}$ is a zero of $\mathbf{x}^{2}+A^{\prime} \mathbf{x}+B^{\prime}=0$ with $\left(A^{\prime}, B^{\prime}\right) \in \Omega$, then $\mathcal{O}_{F}=\mathbb{A}[y]=\mathbb{A}\left[y^{\prime}\right], A=A^{\prime}, y^{\prime}=y+C$ and $B^{\prime}=C^{2}+A C+B$ for some $C \in \mathbb{A}$. The converse is also true.

Lemma 2.2. Let $F=k(y)$ be a quadratic extension of $k$, where $y$ is a zero of $\mathbf{x}^{2}+A \mathbf{x}+B=0$ with $(A, B) \in \Omega$. Then we have

(i) $\infty$ splits in $F$ if and only if $\operatorname{deg}\left(C^{2}+A C+B\right)<2 \operatorname{deg} A$ for some $C \in \mathbb{A}$. In this case, we can always choose $C$ so that $\operatorname{deg}\left(C^{2}+A C+B\right)<\operatorname{deg} A$.

(ii) $\infty$ is inert in $F$ if and only if $\operatorname{deg}\left(C^{2}+A C+B\right)=2 \operatorname{deg} A$ and $\operatorname{sgn}\left(C^{2}+\right.$ $A C+B) \notin \mathcal{P}\left(\mathbb{F}_{q}\right)$ for some $C \in \mathbb{A}$, where $\mathcal{P}(x)=x^{2}+x$ is the ArtinSchreier operator.

(iii) $\infty$ ramifies in $F$ if and only if $\operatorname{deg}\left(C^{2}+A C+B\right)>2 \operatorname{deg} A$ for any $C \in \mathbb{A}$.

Proof. Consider $\mathcal{S}=\left\{\operatorname{deg}\left(C^{2}+A C+B\right): C \in \mathbb{A}\right\}$. We may assume that $\operatorname{deg} B$ is a minimal among the elements in the set $\mathcal{S}$. We will show that

(1) if $\operatorname{deg} B<2 \operatorname{deg} A$, then $\infty$ splits in $F$.

(2) if $\operatorname{deg} B=2 \operatorname{deg} A$ and $\operatorname{sgn}(B) \notin \mathcal{P}\left(\mathbb{F}_{q}\right)$, then $\infty$ is inert in $F$.

(3) if $\operatorname{deg} B=2 \operatorname{deg} A$ and $\operatorname{sgn}(B) \in \mathcal{P}\left(\mathbb{F}_{q}\right)$, then $\operatorname{deg} B$ is not minimal.

(4) if $\operatorname{deg} B>2 \operatorname{deg} A$, then $\infty$ ramifies in $F$.

(1) Suppose that $\operatorname{deg} B<2 \operatorname{deg} A$. Then the equation

$$
\mathbf{z}^{2}+\mathbf{z}+\frac{B}{A^{2}}=0
$$

has two distinct zeros in $k_{\infty}$ by Hensel's Lemma. Put $\mathbf{x}=A \mathbf{z}$. Then the equation

$$
\mathbf{x}^{2}+A \mathbf{x}+B=0
$$

also has two distinct zeros in $k_{\infty}$. Hence $\infty$ splits in $F$.

(2) Suppose that $\operatorname{deg} B=2 \operatorname{deg} A$ and $\operatorname{sgn}(B) \notin \mathcal{P}\left(\mathbb{F}_{q}\right)$. Then

$$
\mathbf{z}^{2}+\mathbf{z}+\frac{B}{A^{2}} \equiv \mathbf{z}^{2}+\mathbf{z}+\operatorname{sgn}(B) \bmod 1 / t
$$

is a separable irreducible polynomial modulo $1 / t$. Hence $\infty$ is inert in $F$.

(3) Suppose that $\operatorname{deg} B=2 \operatorname{deg} A$ and $\operatorname{sgn}(B) \in \mathcal{P}\left(\mathbb{F}_{q}\right)$, say $\operatorname{sgn}(B)=\beta^{2}+\beta$ for some $\beta \in \mathbb{F}_{q}^{*}$. Then $\operatorname{deg}\left((\beta A)^{2}+A(\beta A)+B\right)<\operatorname{deg} B$, so $\operatorname{deg} B$ is not minimal.

(4) Suppose that $\operatorname{deg} B>2 \operatorname{deg} A$. If $\operatorname{deg} B$ is even, say $\operatorname{deg} B=2 n$ and $B=\beta^{2} t^{2 n}+$ lower terms, then $\operatorname{deg}\left(\left(\beta t^{n}\right)^{2}+A\left(\beta t^{n}\right)+B\right)<\operatorname{deg} B$. So $\operatorname{deg} B$ must be odd. Let $\operatorname{deg} B-2 \operatorname{deg} A=2 m+1$. Consider the equation

Put $\mathbf{w}=t^{-m-1} \mathbf{z}$. Then

$$
\mathbf{z}^{2}+\mathbf{z}+\frac{B}{A^{2}}=0 .
$$

$$
\mathbf{w}^{2}+t^{-m-1} \mathbf{w}+t^{-2 m-2} \frac{B}{A^{2}}
$$


is an Eisenstein polynomial at $\infty$. Hence $\infty$ ramifies in $F$.

Remark 2.3. We can give an equivalence relation $\sim$ on the set $\Omega$ as follow;

$$
(A, B) \sim\left(A^{\prime}, B^{\prime}\right) \Leftrightarrow A=A^{\prime} \text { and } B^{\prime}=C^{2}+A C+B \text { for some } C \in \mathbb{A} .
$$

Let $\widetilde{\Omega}$ be the set of equivalence classes with respect to $\sim$. Then we see that there is an one to one correspondence between $\widetilde{\Omega}$ and the set of all quadratic extensions of $k$. We also can show that for any real quadratic extension $F$ of $k$, there is a unique $(A, B) \in \Omega$ such that $\operatorname{deg} B<\operatorname{deg} A$ and $F=k(y)$, where $y$ is a zero of $\mathbf{x}^{2}+A \mathbf{x}+B=0$.

Let $A(t) \in \mathbb{A}$ be one of the following polynomials $t^{2 g}+t^{g}+1, t^{g}+1$ with $g$ odd or $t^{g}+t+1$. It is easy to see that $A$ is square-free. Let $\mathcal{M}_{k}(A)$ be the set of monic polynomials $U \in \mathbb{A}$ of degree $k$ such that $A(U)$ is square-free. Following the same argument as in $[3, \S 2]$ with $A(t)$, we get the following lemma.

Lemma 2.4. $\left|\mathcal{M}_{k}(A)\right| \gg q^{k}$.

Lemma 2.5. Let $g$ be a positive integer. Let $A(t)=t^{g}+t+1 \in \mathbb{A}$ and $\mathcal{M}_{k}(A)$ be the set of monic polynomials $U \in \mathbb{A}$ of degree $k$ such that $A(U)$ is squarefree. For $U, V \in \mathcal{M}_{k}(A)$, if $A(U)=A(V)$, then $U=V$ or $U+V \in \mathbb{F}_{q}^{*}$. Hence there are at most $q$ times repetitions on $A(U)$.

Proof. Suppose $A(U)=A(V)$ with $U, V \in \mathcal{M}_{k}(A)(U \neq V)$. Let $W=U+V$. Then $\operatorname{deg} W<k$. From $A(V)=(U+W)^{g}+(U+W)+1=A(U)$, we get

$$
\sum_{h=0}^{g-1}\left(\begin{array}{l}
g \\
h
\end{array}\right) U^{h} W^{g-h}=W .
$$

Clearly $\operatorname{deg} U^{h_{1}} W^{g-h_{1}}<\operatorname{deg} U^{h_{2}} W^{g-h_{2}}$ for any $0 \leq h_{1}<h_{2} \leq g-1$, since $\operatorname{deg} W<k=\operatorname{deg} U$. Let $n$ be the largest one among $0 \leq h \leq g-1$ such that $\left(\begin{array}{l}g \\ h\end{array}\right) \neq 0$. If $n>0$, then the degree of left hand side in (2.2) is equal to $n k+(g-n) \operatorname{deg} W$, which is greater than $\operatorname{deg} W$. Hence $n=0$ and $W^{g}=W$, so $W \in \mathbb{F}_{q}^{*}$. Therefore, there are at most $q$ times repetitions on $A(U)$.

\section{Proof of Theorem 1.1}

Let $g$ be a positive integer $\geq 2$. Let $U \in \mathbb{A}$ be a monic polynomial,

$$
A= \begin{cases}U^{2 g}+U^{g}+1 & \text { if } g \text { is odd, } \\ U^{g+1}+1 & \text { if } g \text { is even, }\end{cases}
$$

and $B=U^{g}$. Let $y$ satisfy the equation $\mathbf{x}^{2}+A \mathbf{x}+B=0$. Then $F=k(y)$ is a real quadratic extension of $k$ by Lemma 2.2 .

Lemma 3.1. Let $A, B, y$ be as above. If $A$ is square-free, then $\mathcal{O}_{F}=\mathbb{A}[y]$. 
Proof. By Lemma 2.1, we need to show that for any irreducible divisor $P$ of $A$, the congruence $(2.1)$ has no solution in $\mathbb{A}$. Suppose that $D$ is a solution of (2.1). First consider the case that $g$ is odd, so $A=U^{2 g}+U^{g}+1$. Since $P \mid A=B^{2}+B+1$, we have $D \equiv B+1 \bmod P$. Then

$$
(B+1)^{2}+A(B+1)+B \equiv 0 \bmod P^{2} .
$$

But

$(B+1)^{2}+A(B+1)+B=A(B+1)+\left(B^{2}+B+1\right)=A(B+1)+A=A B$, which cannot be divisible by $P^{2}$ since $A$ is square-free and $P \nmid B$, and we get a contradiction.

Now, we consider the case that $g$ is even, so $A=U^{g+1}+1$. Then $D \equiv$ $U^{g / 2} \bmod P$, so

$$
0 \equiv D^{2}+A D+B \equiv A U^{g / 2} \bmod P^{2},
$$

which is impossible since $A$ is square-free and $P \nmid U$.

Lemma 3.2. Let $A, B, y$ be as above. If $A$ is square-free, then the ideal class group of $\mathcal{O}_{F}$ contains an element of order $g$.

Proof. From a straightforward computation, the continued fraction of $y$ is

$$
\begin{cases}{[\overline{A: B+1, B+1}]} & \text { if } g \text { is odd, } \\ {[\overline{A: U, A /(U+1), U}]} & \text { if } g \text { is even, }\end{cases}
$$

and

$$
\begin{cases}q_{3 i}=1, q_{3 i+1}=q_{3 i+2}=U^{g} & \text { if } g \text { is odd, } \\ q_{4 i}=1, q_{4 i+1}=q_{4 i+3}=U^{g}, q_{4 i+2}=U+1 & \text { if } g \text { is even, }\end{cases}
$$

where $q_{h}$ is the denominator of $h$-th iterate of $y$. Now

$$
\mathcal{N}(y)=y(y+A)=B=U^{g},
$$

where $\mathcal{N}$ is the norm map from $F$ to $k$. Let $U=\prod_{i} P_{i}^{e_{i}}$. Since

$$
\mathbf{x}^{2}+A \mathbf{x}+B \equiv \mathbf{x}^{2}+\mathbf{x} \equiv \mathbf{x}(\mathbf{x}+1) \bmod P_{i},
$$

$P_{i}$ splits in $F$. Say $P_{i} \mathcal{O}_{F}=\mathfrak{P}_{i} \mathfrak{P}_{i}^{\prime}$. Since $P_{i} \mid y$, choose $\mathfrak{P}_{i} \mid y$. Then $\mathfrak{P}_{i}^{e_{i} g}|| y$, and $y \mathcal{O}_{F}=\prod_{i} \mathfrak{P}_{i}^{e_{i} g}$. Let $\mathfrak{A}=\prod_{i} \mathfrak{P}_{i}^{e_{i}}$. Then as in [4], we see that $\mathcal{N}(\mathfrak{A})=\alpha U$ with $\alpha \in \mathbb{F}_{q}^{*}$.

Suppose that $\mathfrak{A}^{r}$ is principal for some $r<g$. Then

$$
\left\|\mathcal{N}\left(\mathfrak{A}^{r}\right)\right\|=\|U\|^{r}<\|U\|^{g}<\|A\|,
$$

where we use the same $\mathcal{N}$ for the norm map on ideals. Applying Lemma 5.4 in [1], we have $\mathcal{N}\left(\mathfrak{A}^{r}\right)=\beta q_{i}$ for some $i \geq 0$ with $\beta \in \mathbb{F}_{q}^{*}$. Since $q_{i} \in\left\{1, U^{g}\right\}$ or $q_{i} \in\left\{1, U+1, U^{g}\right\}$ according as $g$ is even or odd, and $\mathcal{N}(\mathfrak{A})=\alpha U$, we get a contradiction. So the order of the ideal class of $\mathfrak{A}$ is $g$. 
Let $A(t) \in \mathbb{A}$ be $t^{2 g}+t^{g}+1$ or $t^{g+1}+1$ according as $g$ is odd or even. By Lemma 2.4, there are $\gg q^{k}$ monic polynomials $U \in \mathbb{A}$ of degree $k$ such that $A(U)$ is square-free. Now we check the repetitions on $A(U)$. It is easy to see that for $U, V \in \mathcal{M}_{k}(A)$, we have

$$
A(U)=A(V) \Leftrightarrow \begin{cases}U=V \text { or } U^{g}+V^{g}=1 & \text { if } g \text { is odd, } \\ U=V & \text { if } g \text { is even. }\end{cases}
$$

Moreover, when $g$ is odd, we can see that for $U, V, W \in \mathcal{M}_{k}(A), U^{g}+V^{g}=$ $U^{g}+W^{g}=1$ holds only if $V=W$. So there are at most double repetitions on $A(U)$. Thus there are $\gg q^{\nu(g, \ell)}$ monic square-free polynomials $A(U)$ with $\operatorname{deg} A(U) \leq \ell$, where $\nu(g, \ell)$ is $\frac{\ell}{2 g}$ or $\frac{\ell}{g+1}$ according as $g$ is odd or even. By Lemma 3.2, the corresponding real quadratic function fields $F=k(y)$ have elements of order $g$ in their ideal class groups. We remark that distinct choice of $A(U)$ gives rise to distinct real quadratic extension $F=k(y)$. This completes the proof of Theorem 1.1.

\section{Proof of Theorem 1.2}

Let $g$ be a positive integer $\geq 2$. Let $U \in \mathbb{A}$ be a monic polynomial,

$$
A= \begin{cases}U^{g}+1 & \text { if } g \text { is odd, } \\ U^{g}+U+1 & \text { if } g \text { is even, }\end{cases}
$$

and $B=\gamma U^{2 g}$, where $\gamma \in \mathbb{F}_{q} \backslash \mathcal{P}\left(\mathbb{F}_{q}\right)$ with $\mathcal{P}(x)=x^{2}+x$. Let $y$ satisfy the equation $\mathbf{x}^{2}+A \mathbf{x}+B=0$. Then, by Lemma 2.2 , we see that $F=k(y)$ is an inert imaginary quadratic extension of $k$.

Lemma 4.1. Let $A, B, y$ be as above. If $A$ is square-free, then $\mathcal{O}_{F}=\mathbb{A}[y]$.

Proof. We have to show that for any irreducible polynomial $P$ dividing $A$, the congruence (2.1) is not solvable in $\mathbb{A}$. Suppose that $D$ is a solution of $(2.1)$. Then $D \equiv \beta U^{g} \bmod P$ for $\beta \in \mathbb{F}_{q}^{*}$ with $\beta^{2}=\gamma$. Then

$$
0 \equiv D^{2}+A D+B \equiv \beta U^{g} A \bmod P^{2},
$$

which is impossible, since $A$ is square-free and $(A, U)=1$.

Lemma 4.2. Let $A, B, y$ be as above. If $A$ is square-free, then the ideal class group of $\mathcal{O}_{F}$ contains an element of order $g$.

Proof. Note that $\mathcal{N}(y)=y(y+A)=B=\gamma U^{2 g}$. Let $U=\prod_{i} P_{i}^{e_{i}}$. Since

$$
\mathrm{x}^{2}+A \mathbf{x}+B \equiv \mathbf{x}^{2}+\mathbf{x} \equiv \mathbf{x}(\mathbf{x}+1) \bmod P_{i},
$$

$P_{i}$ splits in $F$. Choose a prime ideal $\mathfrak{P}_{i}$ of $\mathcal{O}_{F}$ lying over $P_{i}$ such that $\mathfrak{P}_{i} \mid y$. Let $\mathfrak{A}=\prod_{i} \mathfrak{P}_{i}^{e_{i}}$. Then $\mathfrak{A}^{2 g}=y \mathcal{O}_{F}$ and $\mathfrak{A}^{\prime 2 g}=(y+A) \mathcal{O}_{F}$. As before, $\mathcal{N}(\mathfrak{A})=\alpha U$ with $\alpha \in \mathbb{F}_{q}^{*}$.

Suppose that $\mathfrak{A}^{r}$ is principal for some $r<g$, say $\mathfrak{A}^{r}=(C+D y)$. Then

$$
q^{r \operatorname{deg} U}=\left\|\mathcal{N}\left(\mathfrak{A}^{r}\right)\right\|=\|\mathcal{N}(C+D y)\|=\left\|C^{2}+A C D+B D^{2}\right\|,
$$


since $N(C+D y)=(C+D y)(C+D(y+A))=C^{2}+A C D+B D^{2}$. Since $r<g$, we must have $\operatorname{deg} C^{2}=\operatorname{deg} B D^{2}$ or $\operatorname{deg} A C D=\operatorname{deg} B D^{2}$ or $\operatorname{deg} C^{2}=\operatorname{deg} A C D$. In any case $\operatorname{deg} C=\operatorname{deg} D U^{g}=\operatorname{deg} D+g \operatorname{deg} U$. Furthermore, let $c$ and $d$ be the leading coefficients of $C$ and $D$, respectively. Then we must have $c^{2}+c d+\gamma d^{2}=0$, which implies that $\gamma=\mathcal{P}(c / d)$, contradicting the choice of $\gamma$. Thus, $g \leq r \mid 2 g$, and so $r$ is divisible by $g$. Then the ideal class of $\mathfrak{A}$ or $\mathfrak{A}^{2}$ is of order $g$.

Let $A(t) \in \mathbb{A}$ be $t^{g}+1$ or $t^{g}+t+1$ according as $g$ is odd or even. By Lemma 2.4, there are $\gg q^{k}$ monic polynomials $U$ of degree $k$ such that $A(U)$ is square-free. Now we check the repetitions on $A(U)$. When $g$ is odd, it can be easily shown that for $U, V \in \mathcal{M}_{k}(A), A(U)=A(V)$ if and only if $U=V$. So, by Lemma 2.5 , there are at most $q$ times repetitions on $A(U)$. Thus there are $\gg q^{\frac{\ell}{g}}$ monic square-free polynomials $A(U)$ with $\operatorname{deg} A(U) \leq \ell$. By Lemma 4.2 , the corresponding inert imaginary quadratic extensions $F=k(y)$ have an element of order $g$ in their ideal class groups. We remark that distinct choice of $A(U)$ give rise to distinct inert imaginary quadratic extension $F=k(y)$. This completes the proof of Theorem 1.2.

\section{Proof of Theorem 1.3}

Let $g$ be a positive integer $\geq 2$. Let $U \in \mathbb{A}$ be a monic polynomial, $A=$ $U^{g-1}+U+1$ and $B=U^{2 g-1}+U^{g}+U^{4}+U^{3}+U^{2}$. Let $y$ satisfy the equation $\mathbf{x}^{2}+A \mathbf{x}+B=0$, and $F=k(y)$. For any $C \in \mathbb{A}$, we have that $\operatorname{deg}\left(C^{2}+A C+\right.$ $B)=\operatorname{deg} C^{2}>2 \operatorname{deg} A$ if $\operatorname{deg} C>\operatorname{deg} A, \operatorname{and} \operatorname{deg}\left(C^{2}+A C+B\right)=\operatorname{deg} B>$ $2 \operatorname{deg} A$ if $\operatorname{deg} C \leq \operatorname{deg} A$. Hence, by Lemma 2.2 , we see that $F=k(y)$ is a ramified imaginary quadratic extension of $k$.

Lemma 5.1. Let $A, B, y$ be as above. If $A$ is square-free, then $\mathcal{O}_{F}=\mathbb{A}[y]$.

Proof. We have to show that for any irreducible polynomial $P$ dividing $A$, the congruence $(2.1)$ is not solvable in $\mathbb{A}$. Suppose that $D$ is a solution of (2.1). Since

$$
B \equiv U(U+1)^{2}+U(U+1)+U^{4}+U^{3}+U^{2} \equiv U^{4} \bmod P,
$$

we see that $D \equiv U^{2} \bmod P$. Then

$$
\begin{aligned}
0 \equiv D^{2}+A D+B & \equiv A U^{2}+U^{2 g-1}+U^{g}+U^{3}+U^{2} \\
& \equiv A^{2} U+A U^{2}+A U \equiv A\left(U^{2}+U\right) \bmod P^{2},
\end{aligned}
$$

which is impossible, since $A$ is square-free and $P \nmid\left(U^{2}+U\right)$.

Lemma 5.2. Let $A, B, y$ be as above and assume that $\operatorname{deg} U$ is odd. If $A$ is square-free, then the ideal class group of $\mathcal{O}_{F}$ contains an element of order $g$.

Proof. Note that $\mathcal{N}\left(y+U^{g}+U^{2}\right)=U^{2 g}$. Let $U=\prod_{i} P_{i}^{e_{i}}$. Since

$$
\mathbf{x}^{2}+A \mathbf{x}+B \equiv \mathbf{x}^{2}+\mathbf{x} \equiv \mathbf{x}(\mathbf{x}+1) \bmod P_{i},
$$


$P_{i}$ splits in $F$. Choose a prime ideal $\mathfrak{P}_{i}$ of $\mathcal{O}_{F}$ lying over $P_{i}$ such that $\mathfrak{P}_{i} \mid(y+$ $\left.U^{g}+U^{2}\right)$. Let $\mathfrak{A}=\prod_{i} \mathfrak{P}_{i}^{e_{i}}$. Then $\mathfrak{A}^{2 g}=\left(y+U^{g}+U^{2}\right) \mathcal{O}_{F}$ and $\mathfrak{A}^{\prime 2 g}=$ $\left(y+U^{g}+U^{2}+A\right) \mathcal{O}_{F}$. As before, $\mathcal{N}(\mathfrak{A})=\alpha U$ with $\alpha \in \mathbb{F}_{q}^{*}$.

Suppose that $\mathfrak{A}^{r}$ is principal for some $r<g$, say $\mathfrak{A}^{r}=(C+D y)$. Then

$$
q^{r \operatorname{deg} U}=\left\|\mathcal{N}\left(\mathfrak{A}^{r}\right)\right\|=\|\mathcal{N}(C+D y)\|=\left\|C^{2}+A C D+B D^{2}\right\|,
$$

since $N(C+D y)=(C+D y)(C+D(y+A))=C^{2}+A C D+B D^{2}$. Since $r<g$, we must have (1) $\operatorname{deg} C^{2}=\operatorname{deg} B D^{2}$ or (2) $\operatorname{deg} A C D=\operatorname{deg} B D^{2}$ or (3) $\operatorname{deg} C^{2}=\operatorname{deg} A C D$. The case (1) cannot happen, since we assumed that $\operatorname{deg} U$ is odd. In case (2), we have $\operatorname{deg} C=g \operatorname{deg} U+\operatorname{deg} D$, and so $\operatorname{deg} C^{2}>\operatorname{deg} A C D=\operatorname{deg} B D^{2}>r \operatorname{deg} U$, which contradicts to (5.1). In case (3), we have $\operatorname{deg} C=(g-1) \operatorname{deg} U+\operatorname{deg} D$. Then $\operatorname{deg} B D^{2}>\operatorname{deg} C^{2}$, and we get a contradiction to (5.1). Thus, $g \leq r \mid 2 g$, and so $r$ is divisible by $g$. Then the ideal class of $\mathfrak{A}$ or $\mathfrak{A}^{2}$ is of order $g$.

Let $A(t)=t^{g-1}+t+1 \in \mathbb{A}$. By Lemma 2.4 , there are $\gg q^{k}$ monic polynomials $U$ of degree $k$ such that $A(U)$ is square-free. By Lemma 2.5, there are at most $q$ times repetitions on $A(U)$. Thus there are $\gg q^{\frac{\ell}{g-1}}$ monic squarefree polynomials $A(U)$ with $\operatorname{deg} A(U) \leq \ell$. By Lemma 5.2, the corresponding ramified imaginary quadratic extensions $F=k(y)$ have an element of order $g$ in their ideal class groups. We remark that distinct choice of $A(U)$ give rise to distinct ramified imaginary quadratic extension $F=k(y)$. This completes the proof of Theorem 1.3.

\section{References}

[1] S. Bae, Real quadratic function fields of Richaud-Degert type with ideal class number one, Proc. Amer. Math. Soc. 140 (2012), no. 2, 403-414.

[2] D. A. Cardon and M. R. Murty, Exponents of class groups of quadratic function fields over finite fields, Canad. Math. Bull. 44 (2001), no. 4, 398-407.

[3] K. Chakraborty and A. Mukhopadhyay, Exponents of class groups of real quadratic function fields, Proc. Amer. Math. Soc. 132 (2004), no. 7, 1951-1955.

[4] C. Friesen, Class number divisibility in real quadratic function fields, Canad. Math. Bull. 35 (1992), no. 3, 361-370.

[5] R. Murty, Exponents of class groups of quadratic fields, Topics in number theory (University Park, PA, 1997), 229-239, Math. Appl., 467, Kluwer Acad. Publ., Dordrecht, 1999.

[6] R. J. Zuccherato, The continued fraction algorithm and regulator for quadratic function fields of characteristic 2, J. Algebra 190 (1997), no. 2, 563-587.

SUNGHAN BAE

Department of Mathematics

KAIST

TAEJON 305-701, KoreA

E-mail address: shbae@kaist.ac.kr 
HWANYUP JUNG

Department of Mathematics Education

Chungbuk National University

Cheonguu 361-763, Korea

E-mail address: hyjung@chungbuk.ac.kr 